\title{
Identification of Major Phenolic Compounds of Chinese Water Chestnut and their Antioxidant Activity
}

\author{
Yanli You ${ }^{1,3}$, Xuewu Duan ${ }^{1}$, Xiaoyi Wei ${ }^{1}$, Xinguo Su ${ }^{1}$, Mouming Zhao ${ }^{2}$, Jian Sun ${ }^{1,3}$, \\ Neungnapa Ruenroengklin ${ }^{1,3}$ and Yueming Jiang ${ }^{1, *}$
}

${ }^{1}$ South China Botanical Garden, The Chinese Academy of Sciences, Guangzhou Leyiju 510650; P. R. China; E-mail: yanliyou2002@163.com

${ }^{2}$ College of Light Industry and Food Science, South China University of Technology, Guangzhou 510640; P. R. China; E-mail: femmzhao@scut.edu.cn

${ }^{3}$ Graduate School, Chinese Academy of Sciences, Beijing 100039; P. R. China

* Author to whom correspondence should be addressed; E-mail: ymjiang@scbg.ac.cn; Tel.: (+86) 20 37252525; Fax: (+86) 2037252831

Received: 7 April 2007; in revised form: 20 April 2007 / Accepted: 23 April 2007 / Published: 25 April 2007

\begin{abstract}
Chinese water chestnut (CWC) is one of the most popular foods among Asian people due to its special taste and medical function. Experiments were conducted to test the antioxidant activity and then determine the major phenolic compound components present in CWC. CWC phenolic extract strongly inhibited linoleic acid oxidation and exhibited a dose-dependent free-radical scavenging activity against $\alpha, \alpha$-diphenyl- $\beta$-picrylhydrazyl (DPPH) radicals, superoxide anions and hydroxyl radicals, which was superior to ascorbic acid and butylated hydroxytoluene (BHT), two commercial used antioxidants. Furthermore, the CWC extract was found to have a relatively higher reducing power, compared with BHT. The major phenolic compounds present in CWC tissues were extracted, purified and identified by high-performance liquid chromatograph (HPLC) as (-)-gallocatechin gallate, $(-)$-epicatechin gallate and (+)-catechin gallate. This study suggests that CWC tissues exhibit great potential for antioxidant activity and may be useful for their nutritional and medicinal functions.
\end{abstract}

Keywords: Chinese water chestnut, phenolic compounds, antioxidant activity, reducing power, free radicals. 


\section{Introduction}

Excessive free radical production and lipid peroxidation are involved in the pathogenesis of some chronic diseases, including atherosclerosis, cardiac and cerebral ischemia, neurodegenerative disorders, carcinogenesis, diabetes, and rheumatic disorders [1-4]. They also play a major role in the aging process [5,6]. In recent years, there has been an increased interest in phenolic compounds derived from fruits and vegetables for their possible health benefits. The anticarcinogenic, antimutagenic, and cardioprotective effects of phenolic compounds are reported to be generally associated with their antioxidant properties of eliminating free radicals and alleviating lipid peroxidation [7]. The antioxidative properties of phenolics mainly arise from their high reactivity as hydrogen or electron donors and from the ability of the polyphenol-derived radicals to stabilize and delocalize the unpaired electron or from their ability to chelate transition metal ions (i.e. cause termination of the Fenton reaction) $[8,9]$.

Chinese water chestnut (Eleocharis tuberosa) is one of the most popular hydrophytic vegetables in Asia because of its unique taste [10]. It is estimated that the annual production of Chinese water chestnut in China was about 600,000 tons in 2006. Chinese water chestnut has also been used as a folk medicine to treat hypertension, chronic nephritis, constipation, pharyngitis, laryngitis and enteritis. Liu et al. isolated a functional compound from Chinese water chestnut (CWC), 24-ethylcholesta$\Delta^{7}$-cholesterol, which had obvious in vivo and in vitro antimicrobial effects on bacteria, inhibited many kinds of inflammation and showed visible activity against pain caused by acetic acid [11]. Furthermore, the addition of functional extracts of CWC in cake reduced the development of oxidation and decay [12]. However, little information is available on the phenolic compound constituents present in CWC and their antioxidant activity.

The objective of the current study was to evaluate the antioxidant property of phenolics from CWC, including inhibition of lipid peroxidation, reducing power and scavenging activities against DPPH radical, superoxide anion and hydroxyl radical. Moreover, the major phenolic compounds from CWC tissues were also identified by HPLC.

\section{Results and Discussion}

\section{Extraction and purification of phenolics from CWC}

In this study, an aqueous acetic acid-acetonitrile gradient and a $\mathrm{C}_{18}$ column were used for isolation of phenolic compounds to obtain good resolution. A typical HPLC profile of the phenolic extract of CWC is shown in Figure 1. No interfering peaks were noted for CWC samples. Peaks 1, 2 and 3 were identified, as (-)-gallocatechin gallate, (-)-epicatechin gallate and (+)-catechin gallate, respectively (Figure 2), according to their spectral characteristics and retention times in comparison with commercial pure compounds. These major phenolic compounds contributed more than $60 \%$ of the total phenolic content (2.53 $\mathrm{mg} / \mathrm{g}$ on fresh weight basis). 
Figure 1. HPLC profile of CWC extract at $280 \mathrm{~nm}$. Peak 1: (-)-gallocatechin gallate; peak 2: (-)-epicatechin gallate and peak 3: $(+)$-catechin gallate.

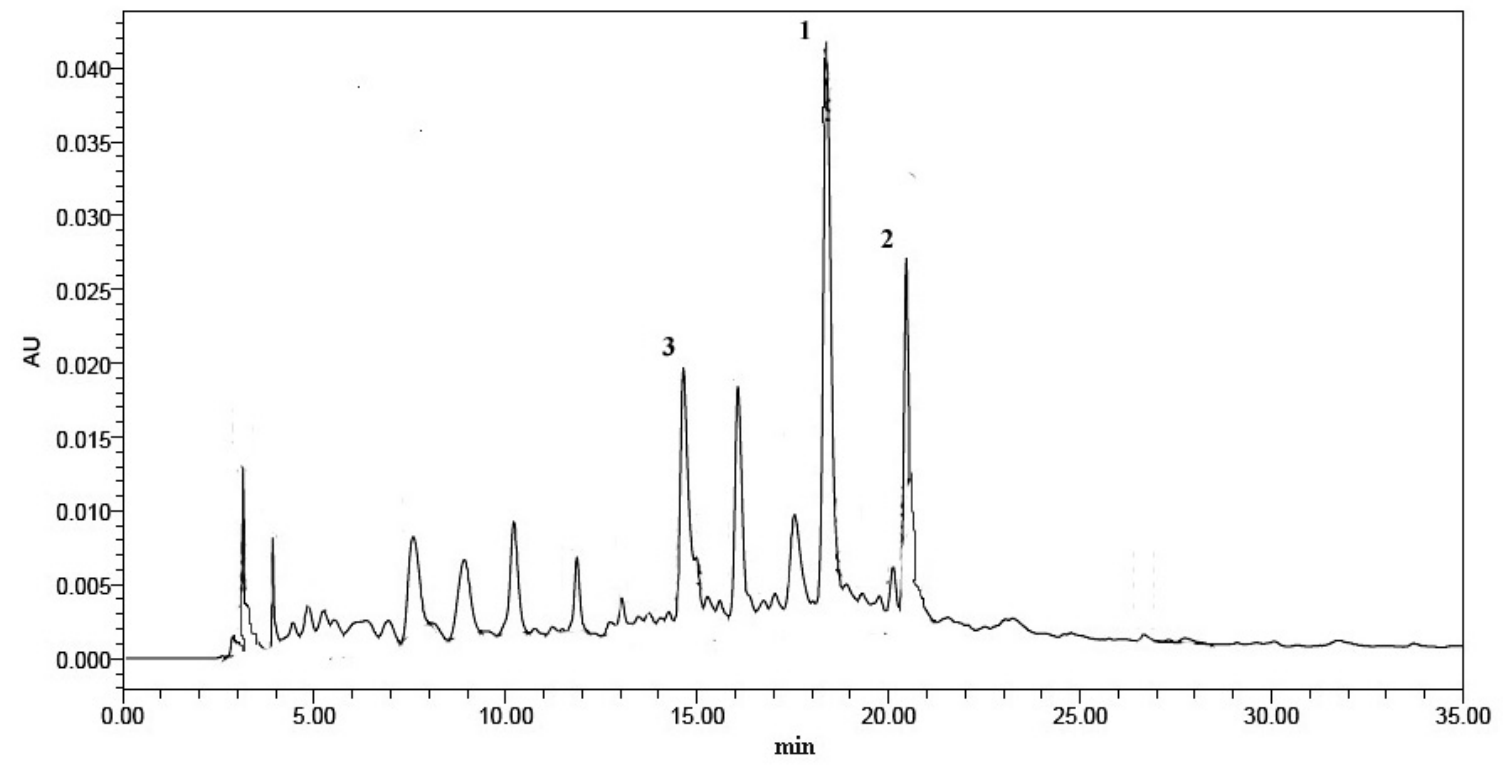

Figure 2. Three major phenolic compounds from CWC tissues by HPLC.<smiles>[CH][C@]1(OC(=O)c2cc(O)c(O)c(O)c2)Cc2c(O)cc(O)cc2O[C@H]1c1cc(O)c(O)c(O)c1</smiles>

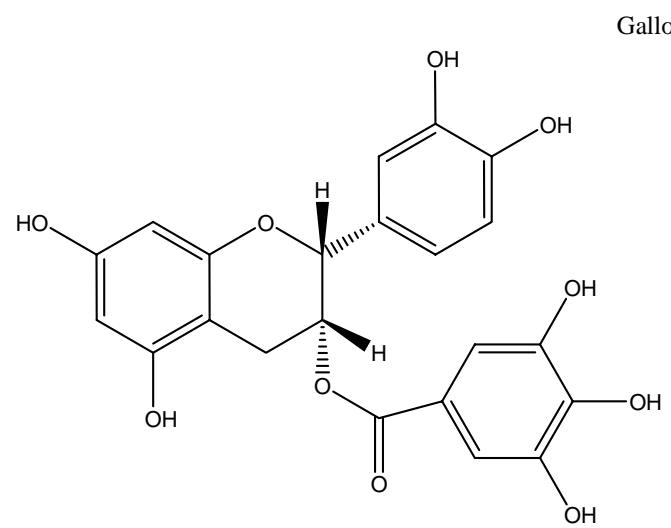

Epicatechin gallate

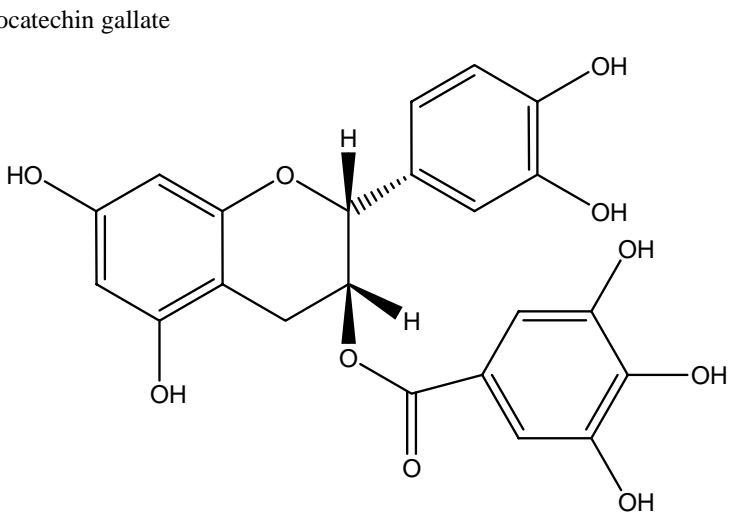

Catechin gallate

Antioxidant activity in linoleic acid system

Polyunsaturated fatty acids, such as linoleic acid, are easily oxidized in the presence of oxygen in the air. This auto-oxidation leads to the occurrence of chain reactions with the formation of conjugated double bonds [13]. The antioxidant effects of the CWC phenolic extract, ascorbic acid and BHT on the 
peroxidation of linoleic acid are presented in Figure 3. Phenolics from CWC can significantly inhibit peroxidation of linoleic acid and reduce the formation of hydroperoxides, which implied that these phenolics are powerful natural antioxidants. Moreover, the measured antioxidant activity of the CWC phenolic extract was significantly higher than that of ascorbic acid, a widely used commercial antioxidant. This strong antioxidant activity of the CWC extract could be due to the structures of the three major phenolic compounds [9].

Figure 3. Antioxidant activity of phenolics from CWC tissues, measured by the ferric thiocyanate method.

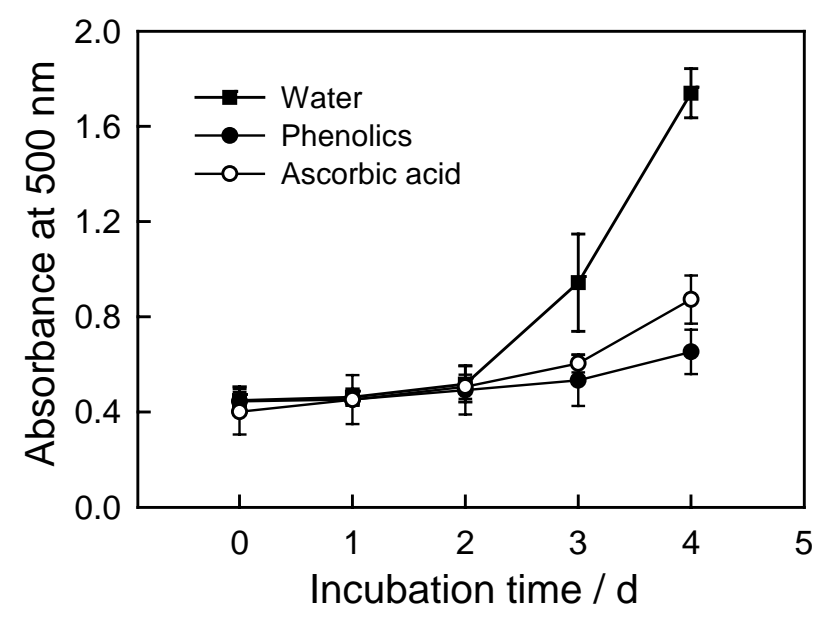

Reducing power

Reducing power is widely used in evaluating the antioxidant activity of polyphenols [14-16]. The reducing property is generally associated with the presence of reductones which exert antioxidant action by breaking the free radical chains via hydrogen atom donation [17]. Reductones are also reported to prevent peroxide formation by reacting with certain precursors of peroxide [18].

Figure 4. Reducing power of phenolics from CWC tissues.

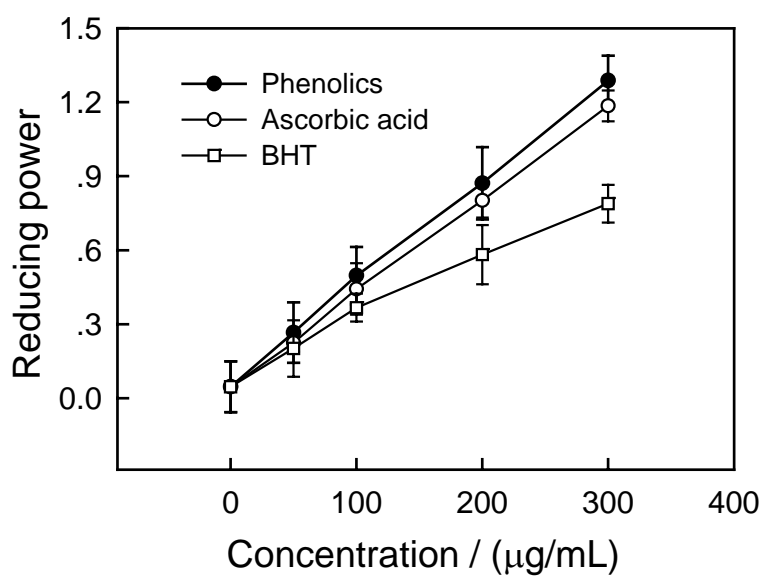

As shown in Figure 4, CWC phenolic extract exhibited higher reducing power than BHT and similar reducing power to ascorbic acid, suggesting that the CWC phenolic extract possesses a strong electron 
donating capacity. Furthermore, a linear relationship existed between the concentration and reducing power of CWC phenolic extract, ascorbic acid and BHT, with correlation coefficients of 0.9995 ( $\mathrm{y}=$ $0.0041 \mathrm{x}+0.0608), 0.9998(\mathrm{y}=0.0038 \mathrm{x}+0.9995)$ and $0.9946(\mathrm{y}=0.0024 \mathrm{x}+0.0795)$ for phenolic extract, ascorbic acid and BHT, respectively. Luximon-Ramma et al. have reported the existence of apparent linear relationships between antioxidant capacity (assessed as ferric reducing power) and total phenol content in Cassia fistula extracts [16]. Similar findings have been obtained in assessing antioxidant activities of crude extracts of Ribes, Rubus and Vaccinium [19], fruit juices [20] and red wine [21].

\section{$D P P H \cdot$ radical scavenging activity}

Free radical scavenging is one of the known mechanisms by which antioxidants inhibit lipid peroxidation [9,22]. The DPPH' radical scavenging activity has been extensively used for screening antioxidants from fruit and vegetable juices or their extracts [23]. Figure 5 shows the DPPH radical scavenging activity of CWC phenolics, ascorbic acid and BHT. The CWC phenolics showed a potent and concentration-dependent free radical scavenging activity against DPPH'radical. Furthermore, the CWC phenolic extract had the highest $\mathrm{DPPH}^{`}$ radical scavenging activity, compared with ascorbic acid and BHT, which might be attributed to the structure of these phenolic compounds [9]. At $100 \mu \mathrm{g} / \mathrm{mL}$, the DPPH' radical scavenging activities of CWC phenolics, ascorbic acid and BHT were 94.4, 73.3 and 40.7\%, respectively. Sun et al. [24] investigated antioxidant properties of 11 different fruits (including cranberry, apple, red grape, strawberry, pineapple, peach, lemon, orange, pear, and grapefruit) using a total oxyradical scavenging capacity assay, and found that there was a direct relationship between total phenolic content and total antioxidant activity in extracts of these fruits.

Figure 5. Scavenging activity of phenolics from CWC tissues on DPPH radical.

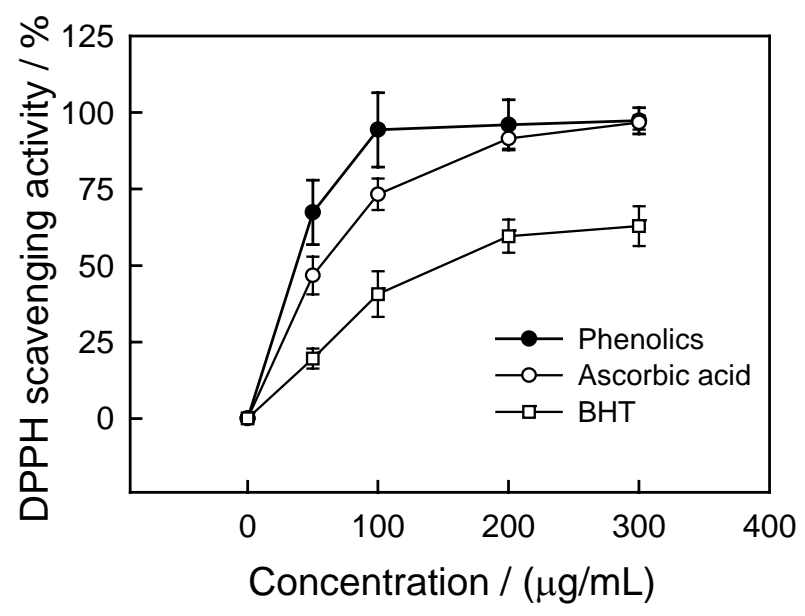

Superoxide anion scavenging activity

Superoxide anion radical is produced by a number of cellular reactions including various enzyme systems, such as lipoxygenases, peroxidase, NADPH oxidase and xanthine oxidase. Superoxide anion radical plays an important role in plant tissues and is involved in the formation of other cell-damaging free radicals [22]. The relative scavenging activity of CWC phenolics on superoxide radical is shown in 
Figure 6. At $100 \mu \mathrm{g} / \mathrm{mL}$ CWC phenolics appeared to be more potent than the positive controls, ascorbic acid and BHT, with their superoxide anion radical scavenging activities being 23.8, 3.4 and 4.6\%, respectively.

Figure 6. Superoxide radical scavenging activity of phenolics from CWC tissues.

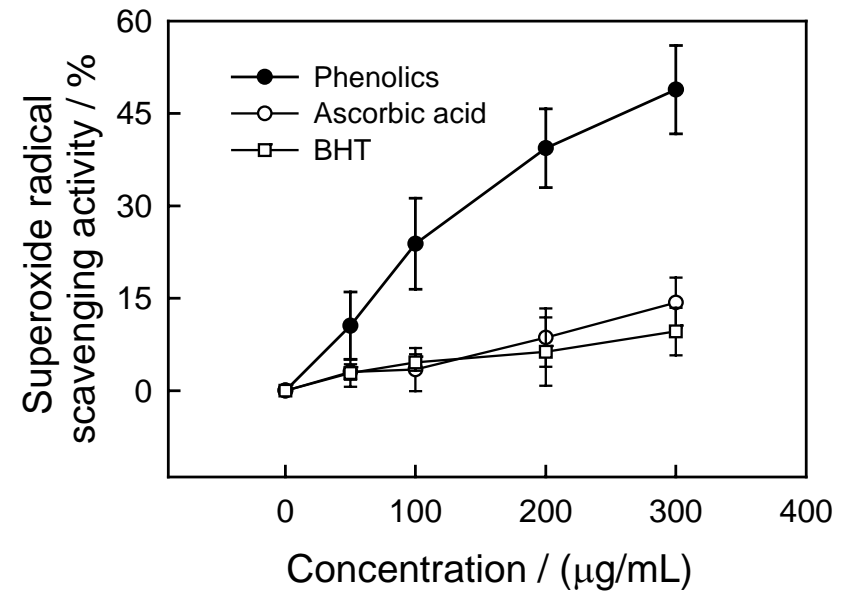

Hydroxyl radical scavenging activity

Hydroxyl radicals generated in the presence of reduced transition metals such as $\mathrm{Fe}^{2+}$ and $\mathrm{H}_{2} \mathrm{O}_{2}$ by the Fenton reaction are known to be the most reactive free radical species, capable of damaging almost every biological molecule found in living cells [25]. These radicals have the capacity to join the nucleotides in DNA and cause strand breakage, which contributes to carcinogenesis, mutagenesis and cytotoxicity [26]. In addition, these species are considered to be rapid initiators of the lipid peroxidation process, due to abstraction of hydrogen atoms from unsaturated fatty acids [25]. To determine the hydroxyl radical scavenging capacity of CWC phenolics, the effect of CWC phenolics on hydroxyl radical generated by $\mathrm{Fe}^{3+}$ ions was analyzed by evaluating the degree of deoxyribose degradation.

Figure 7. Inhibitory effect of phenolics from CWC tissues on deoxyribose degradation by hydroxyl radical.

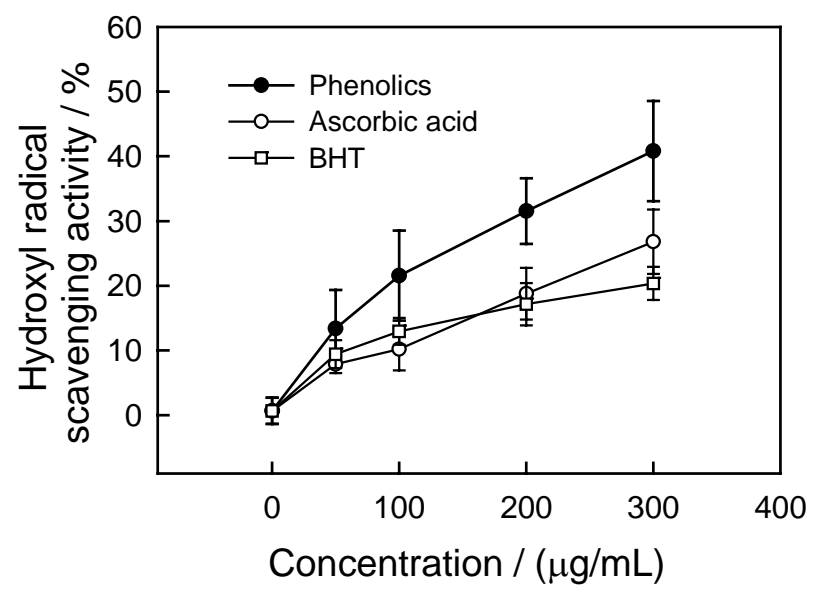


As shown in Figure 7, the concentration-dependent scavenging effect by CWC phenolics and the positive controls, ascorbic acid and BHT, was evident. At concentrations above $50 \mu \mathrm{g} / \mathrm{mL}$, CWC phenolics showed the highest hydroxyl radical scavenging activity, compared with ascorbic acid and BHT. The hydroxyl radical scavenging activities were 31.5, 18.7 and $17.15 \%$ at $200 \mu \mathrm{g} / \mathrm{mL}$ for CWC phenolics, ascorbic acid and BHT, respectively.

\section{Conclusions}

In this study, phenolics were extracted and purified from CWC extract, and three major phenolic components were identified by HPLC as (-)-gallocatechin gallate, (-)-epicatechin gallate and $(+)$-catechin gallate. In a series of in vitro tests, CWC phenolics exhibited strong antioxidant activity. They significantly inhibited the peroxidation of linoleic acid and acted as a strong electron-donating agent in the $\mathrm{Fe}^{3+}$ to $\mathrm{Fe}^{2+}$ assay and hydrogen-donating agents in the $\mathrm{DPPH}$ assay. Furthermore, the phenolics from CWC were effective in scavenging superoxide anion radical and inhibiting deoxyribose degradation induced by hydroxyl radical. The strong antioxidant activity of CWC phenolics may therefore be useful for their food nutrition and medicinal functions.

\section{Experimental Section}

\section{Plant materials}

Fresh Chinese water chestnut (Eleocharis tuberosa) cv. Guilin was obtained from a commercial market in Guangzhou, China. Fruits were selected for uniformity and size, while bruised or diseased fruits were discarded. Fruits were washed, peeled using a sharp stainless steel knife and the phenolic compounds extracted.

\section{Extraction and purification of phenolics}

Phenolic compounds were extracted and purified according to Zhang et al. [27], with some modifications. Fresh CWC tissues (1,000 g) were cut into small pieces, freeze-dried, and then extracted for $10 \mathrm{~h}$ by stirring at $4{ }^{\circ} \mathrm{C}$ with cold $95 \%$ ethanol $(600 \mathrm{~mL})$. The homogenate was filtered through four layers of cheesecloth and the residue was then extracted with two additional portions of alcohol (200 mL each). The combined filtrate was centrifuged at $7,000 \times \mathrm{g}$ for $15 \mathrm{~min}$ at $4^{\circ} \mathrm{C}$ and the residue was discarded. Ethanol was removed from the supernatant on a rotary evaporator under vacuum at $35^{\circ} \mathrm{C}$, and the pigments were eliminated by two successive extractions with petroleum ether. After the addition of $20 \%$ ammonium sulphate and $2 \%$ metaphosphoric acid to the aqueous phase, phenolic compounds were extracted three times with ethyl acetate. These extracts were combined, evaporated and then dried under vacuum at $35^{\circ} \mathrm{C}$. The residue was re-dissolved in methanol and the methanolic extract was filtered through Gelman Nylon Acrodisc $13(0.45 \mu \mathrm{m})$ and stored at $-20{ }^{\circ} \mathrm{C}$ before identification of major phenolic compounds and analyses of antioxidant properties. 
High-Performance Liquid Chromatography (HPLC) analysis

The chromatographic conditions used were as follows: a Waters 2695 Separation Module high-performance liquid chromatograph (Waters) equipped with Pinnacle $\mathrm{C}_{18}$ column $(250 \times 4.6 \mathrm{~mm}$, 5 $\mu \mathrm{m}$ size particle, Waters, Millford, MA, USA) and a Waters 2478 Dual $\lambda$ Absorbance Detector (Waters) operating at $280 \mathrm{~nm}$ was used for the phenolic compounds. The mobile phase was acetonitrile (A) and acidified water containing $2 \%$ acetic acid. The gradient was as follow: 0 min, 1\% A; 4 min, 1\% A; 38 min, 50\% A; $42 \mathrm{~min}, 80 \% \mathrm{~A}$ and then held for $3 \mathrm{~min}$ before returning to the initial conditions. The flow rate was $1 \mathrm{~mL} / \mathrm{min}$ and the injection volume was $20 \mu \mathrm{L}$. Identification of the phenolic compounds was achieved by comparison with retention times of standards, UV spectra and calculation of UV absorbance ratios after co-injection of samples and standards. Commercial standards were purchased from Sigma Chemicals.

\section{Antioxidant activity in linoleic acid system}

The antioxidant activity of CWC phenolics against lipid peroxidation was measured by the peroxidation of linoleic acid using the ferric thiocyanate method (FTC), as described by Takao et al. [28], with some modifications. A reaction mixture containing $100 \mu \mathrm{g} / \mathrm{mL}$ of phenolic extract $(0.2 \mathrm{~mL})$, linoleic acid emulsion ( $25 \mathrm{mg} / \mathrm{mL}$ in $99 \%$ ethanol, $0.2 \mathrm{~mL}$ ) and $50 \mathrm{mM}$ phosphate buffer $(\mathrm{pH}=7.4,0.4$ $\mathrm{mL})$ was incubated in the dark at $40{ }^{\circ} \mathrm{C}$. An aliquot $(0.1 \mathrm{~mL})$ of the reaction solution was then added to $70 \%(\mathrm{v} / \mathrm{v})$ ethanol $(3 \mathrm{~mL})$ and 30\% (w/v) ammonium thiocyanate $(0.1 \mathrm{~mL})$. After $3 \mathrm{~min}, 20 \mathrm{mmol} / \mathrm{L}$ ferrous chloride in $3.5 \%(\mathrm{v} / \mathrm{v})$ hydrochloric acid $(0.1 \mathrm{~mL})$ was added to the reaction mixture. The absorbance of the resulting solution was measured at $500 \mathrm{~nm}$ using a Unic UV-2802 spectrophotometer. Aliquots were assayed every $24 \mathrm{~h}$ until the absorbance of the water solution (without CWC phenolics) reached the maximum value. Ascorbic acid was used as a positive control.

\section{Reducing power}

The reducing power was determined according to the method of Oyaizu [29].

\section{Scavenging activity of DPPH radical}

The scavenging activity of CWC phenolics against DPPH' radical was assessed according to the method described by Duan et al. [30].

\section{Superoxide radical scavenging activity}

Determination of superoxide radical scavenging activity was conducted according to the method described by Siddhurajua et al. [31]. 


\section{Hydroxyl radical scavenging activity}

The assay was performed as described by Lee et al. [32] with some modifications. An aliquot (0.1 $\mathrm{mL}$ ) of CWC phenolics at different concentrations was mixed with reaction buffer $\left(100 \mu \mathrm{mol} / \mathrm{L} \mathrm{FeCl}_{3}\right.$, $104 \mu \mathrm{mol} / \mathrm{L}$ EDTA, $1.5 \mathrm{mmol} / \mathrm{L} \mathrm{H}_{2} \mathrm{O}_{2}, 2.5 \mathrm{mmol} / \mathrm{L}$ deoxyribose, and $100 \mu \mathrm{mol} / \mathrm{L} \mathrm{L}$-ascorbic acid, $\mathrm{pH}=7.4,1 \mathrm{~mL})$ and then incubated for $1 \mathrm{~h}$ at $37^{\circ} \mathrm{C}$. 2-Thiobarbituric acid $(0.5 \%, 1 \mathrm{~mL})$ was added and the mixture was then heated for $30 \mathrm{~min}$ at $80^{\circ} \mathrm{C}$. The solution was cooled on ice and the absorbance was then measured at $532 \mathrm{~nm}$. The control contained methanol instead of CWC phenolic solution. Percent inhibition of deoxyribose degradation by hydroxyl radical was calculated as $\left(1-A_{\text {sample }} / A_{\text {control }}\right) \times 100$, where $A$ was the absorbance using sample or control. Ascorbic acid and BHT were used as the positive controls.

\section{Total phenolic content}

Total phenolic content in the above-mentioned CWC extract was determined according to the method of Folin-Ciocalteu reaction [33], using gallic acid as the standard.

\section{Statistical analysis}

All analyses were performed in triplicate. The data were expressed as means \pm standard error in five figures and then analyzed by SPSS (Version 10.0). One-way analysis of variance (ANOVA) and Tukey multiple comparisons were carried out to test any significant difference between the means. Differences between means at the $5 \%$ level were considered to be significant.

\section{Acknowledgements}

This work was supported partly by the National Natural Science Foundation of China (Grant Nos. 30425040 and U0631004) and Guangdong Provincial Natural Science Foundation (No. 06200670).

\section{References}

1. Halliwell, B.; Gutteridge, J.M.C.; Cross, C.E. Free radicals, antioxidants and human disease: where are we now? J. Lab. Clin. Med. 1992, 119, 598-620.

2. Lefer, D.J.; Grander, D.N. Oxidative stress and cardiac disease. Amer. J. Med. 2000, 109, 315-323.

3. Viana, M.; Aruoma, O. I.; Herrera, E.; Bonet, B. Oxidative damage in pregnant diabetic rats and their embryo. Free Radical Biol. Med. 2000, 29, 1115-1121.

4. Hanninen, O.; Kaartinen, K.; Rauma, A.L.; Nenenen, M.; Torronen, R.; Hakkinen, S.; Adlercreutz, H.; Laakso, J. Antioxidants in vegan diet and rheumatic disorders. Toxicology 2000, 155, 45-53.

5. Hu, H.L.; Forsey, R.J.; Blades, T. J.; Barott, M.E.J. Antioxidant may contribute in the fight against aging: an in vitro model. Mech. Ageing Dev. 2000, 121, 217-230.

6. Khodr, B.; Khalil, Z. Modulation of inflammation by reactive oxygen species: implications for aging and tissue repair. Free Radical Biol. Med. 2001, 30, 1-8. 
7. Potter, J.D. Vegetables, fruit, and cancer. Lancet 2005, 366, 527-530.

8. Robards, K.; Prenzler, P. D.; Tucker, G.; Swatsitang, P.; Glover, W. Phenolic compounds and their role in oxidative processes in fruit. Food Chem. 1999, 66, 401-436.

9. Rice-Evans, C.; Miller, N.J.; Paganga, G. Antioxidant properties of phenolic compounds. Trends Plant Sci. 1997, 2, 152-159.

10. Peng, L.T.; Jiang, Y.M. Effects of chitosan coating on shelf life and quality of fresh-cut Chinese water chestnut. LWT-Food Sci. Technol. 2003, 36, 359-364.

11. Liu, X.; Zhao, L.C.; Zhou, A.M. Preliminary study on functional component and functional activities of waste slurry derived in processing water chestnut starch. Food Sci. 2006, 27, 251-256.

12. Zhao, L.C.; Liu, X.; Chen, Y.Q. Preliminary study of chufa functional components in preserving water chestnut cake. Food Sci. 2005, 26, 422-424.

13. Stoilova, I.; Krastanov, A.; Stoyanova, A.; Denevc, P.; Gargova, S. Antioxidant activity of a ginger extract (Zingiber officinale). Food Chem. 2007, 102, 764-770.

14. Soong, Y.Y.; Barlow, P.J. Antioxidant activity and phenolic content of selected fruit seeds. Food Chem. 2004, 88, 411-417.

15. Chang, L.W.; Yen, W.J.; Huang, S.C.; Duh, P.D. Antioxidant activity of sesame coat. Food Chem. 2002, 78, 347-354.

16. Luximon-Ramma, A.; Bahorun, T.; Soobrattee, M.A.; Aruoma, O.I. Antioxidant activities of phenolic, proanthocyanidin, and flavonoid compontents in extracts of Cassia fistula. J. Agric. Food Chem. 2002, 50, 5042-5047.

17. Pin-Der-Duh, X. Antioxidant activity of burdock (Arctium lappa Linne): its scavenging effect on free-radical and active oxygen. J. Amer. Oil Chem. Soc. 1998, 75, 455-461.

18. Ratheea, J.S.; Hassarajania, S.A.; Chattopadhyay, S. Antioxidant activity of Nyctanthes arbor-tristis leaf extract. Food Chem. 2007, in press.

19. Deithton, N.; Brnnan, R.; Finn, C.; Davies, H.V. Antioxidant properties of domesticated and wild Rubus species. J. Agric. Food Chem. 2000, 80, 1307-1313.

20. Gil, M.I.; Tomas-Barberan, F.A.; Hess-Pierce, B.; Holcroft, D.M.; Kader, A.A. Antioxidant activity of pomegranate juice and its relationship with phenolic composition and processing. $J$. Agric. Food Chem. 2000, 48, 4581-4589.

21. Burns, J.; Gardner, P.T.; Mcphail, D.B.; O’nell, J.; Crawford, S.; Morecroft, I.; Lister, C.; Matthews, D.; Maclean, M.R.; Lean, M.E.J.; Duthie, G.G.; Crozier, A. Antioxidant activity, vasodilation capacity and phenolic content of red wines. J. Agric. Food Chem. 2000, 48, 220-230.

22. Bloknina, O.; Virolainen, E.; Fagerstedt, K.V. Antioxidants, oxidative damage and oxygen deprivation stress: a Review. Ann. Bot. 2003, 91, 179-194.

23. Sanchez-Moreno, C. Methods used to evaluate the free radical scavenging activity in foods and biological systems. Food Sci. Technol. Int. 2002, 8, 121-137.

24. Sun, J.; Chu, Y.F.; Wu, X.; Liu, R.H. Antioxidant and antiproliferative activities of common fruits. J. Agric. Food Chem. 2002, 50, 7449-7454.

25. Rollet-Labelle, E.; Grange, M.J.; Elbim, C.; Marquetty, C.; Gougerot-Pocidalo, M.A.; Pasquier, C. Hydroxyl radical as a potential intracellular mediator of polymorphonuclear neutrophil apoptosis. Free Radical Bio. Med. 1998, 24, 563-572. 
26. Moskovitz, J.; Yim, K.A.; Choke, P.B. Free radicals and disease. Arch. Biochem. Biophys. 2002, 397, 354-359.

27. Zhang, D.L.; Quantick, P.C.; Grigor, J.M. Changes in phenolic compounds in litchi (Litchi chinensis Sonn.) fruit during posthravest storage. Postharv. Biol. Technol. 2000, 19, 165-172.

28. Takao, T.; Kitatani, F.; Watanabe, N.; Yagi, A.; Sakata, K. A simple screening method for antioxidants and isolation of several antioxidants produced by marine bacteria from fish and shellfish. Biosci. Biotechnol. Biochem. 1994, 58, 1780-1783.

29. Oyaizu, M. Studies on products of browning reaction: antioxidative activity of products of browning reaction prepared from glucosamine. J. Nutr. 1986, 44, 307-315.

30. Duan, X.W.; Jiang, Y.M.; Su, X.G.; Zhang, Z.Q.; Shi, J. Anthioxidant properties of anthocyanins extracted from litchi (Litchi chinenesis Sonn) fruit pericarp tissue in relation to their role in the pericarp browning. Food Chem. 2007, 101, 1382-1388.

31. Siddhurajua, P.; Mohanb, P.S.; Bechera, K. Studies on the antioxidant activity of Indian Laburnum (Cassia fistula L.): a preliminary assessment of crude extracts from stem bark, leaves, flowers and fruit pulp. Food Chem. 2002, 79, 61-67.

32. Lee, J.C.; Kim, H.R.; Kim, J.; Jang, Y.S. Antioxidant property of an ethanol extract of the stem of Opuntia ficus-indica var. Saboten. J. Agric. Food Chem. 2002, 50, 6490-6496.

33. Singleton, V.L.; Rossi, J.A. Colorimetry of total phenolics with phosphomolybdic-phosphotungstic acid reagents. Amer. J. Enol. Viticult. 1965, 16, 144-158.

Sample Availability: Available from the authors.

(C) 2007 by MDPI (http://www.mdpi.org). Reproduction is permitted for noncommercial purposes. 